\title{
Ghost Branch Photoluminescence From a Polariton Fluid Under Nonresonant Excitation
}

\author{
Maciej Pieczarka, ${ }^{1, *}$ Marcin Syperek, ${ }^{1}$ Łukasz Dusanowski, ${ }^{1}$ Jan Misiewicz, ${ }^{1}$ Fabian Langer, ${ }^{2}$ Alfred Forchel, ${ }^{2}$ \\ Martin Kamp, ${ }^{2}$ Christian Schneider, ${ }^{2}$ Sven Höfling, ${ }^{2,3}$ Alexey Kavokin, ${ }^{4,5}$ and Grzegorz Sęk ${ }^{1}$ \\ ${ }^{1}$ Laboratory for Optical Spectroscopy of Nanostructures, Department of Experimental Physics, \\ Wroctaw University of Technology, Wybrzeże Wyspianskiego 27, 50-370 Wrocław, Poland \\ ${ }^{2}$ Technische Physik, University of Würzburg and Wilhelm-Conrad-Röntgen-Research Center \\ for Complex Material Systems (RCCM), Am Hubland, D-97074 Würzburg, Germany \\ ${ }^{3}$ SUPA, School of Physics and Astronomy, University of St. Andrews, St. Andrews KY16 9SS, United Kingdom \\ ${ }^{4}$ Spin Optics Laboratory, Saint Petersburg State University, 1 Ulianovskaya, 198504 St. Petersburg, Russia \\ ${ }^{5}$ Physics and Astronomy School, University of Southampton, Highfield, Southampton SO17 1BJ, United Kingdom
}

(Received 23 March 2015; revised manuscript received 15 July 2015; published 26 October 2015)

An expanding polariton condensate is investigated under pulsed nonresonant excitation with a small laser pump spot. Far above the condensation threshold we observe a pronounced increase in the dispersion curvature, with a subsequent linearization of the spectrum and strong luminescence from a ghost branch orthogonally polarized with respect to the linearly polarized condensate emission. Polarization of both branches is understood in terms of spin-dependent polariton-polariton scattering. The presence of the ghost branch has been confirmed in time-resolved measurements. The effects of disorder and dissipation in the photoluminescence of polariton condensates and their excitations are discussed.

DOI: 10.1103/PhysRevLett.115.186401

Exciton polaritons are composite bosons consisting of strongly coupled microcavity photons and quantumwell excitons. They are able to form a novel class of condensates (for recent reviews see, e.g., [1,2]). Despite their nonequilibrium and dissipative nature, they behave as condensates of weakly interacting bosons. Collective phenomena like condensation [3], off-diagonal long-range order [4,5], topological excitations [6], or superfluid features in the propagation of polariton flows [7] have been demonstrated in such systems. A spectacular consequence of the parametric scattering in polariton gases is the appearance of nonparabolic scattering bands, including normal branches (NBs) and ghost branches (GBs), where the latter are populated by the virtual off-branch excitonpolaritons $[7,8]$. Excitations of polariton condensates are expected to be characterized by linearized dispersions of the Bogoliubov-like spectra [9]. The fluid excitations at low momenta are expected to behave as collective sound waves rather than as single particles. Recently, the linear dispersion NB and the GB of a resonantly pumped polariton condensate [10] have been observed in a four-wave mixing experiment.

The experiment providing direct access to the dispersion of excitations of spontaneously formed condensates in polariton systems is photoluminescence (PL) under nonresonant excitation. The nonresonant pumping can be realized with either a detuned laser or the current injection $[11,12]$. In the nonresonant pumping scheme, incoherent excitons are generated and then relax, subsequently feeding the reservoir and governing the dynamics of the system [13]. The relaxation of the created hot excitons involves multiple-scattering processes, which destroy the coherence
PACS numbers: 71.36.+c, 67.10.-j, 71.35.Lk, 78.67.De

and phase of the excitation; this ensures that these properties are not inherited by the condensate, in contrast to the resonant excitation case. At the same time, the incoherent reservoir causes additional decoherence [14], forming a repulsive potential [15] which shapes the condensate spatially and spectrally. Moreover, it significantly affects the excitation spectrum of the condensate [16]. Bogoliubov dispersions have been reported in this excitation scheme; however, no fingerprints of the GB have been observed yet $[17,18]$. This has fueled the debate on the possibility of observing the GB in nonresonant PL [19], where the strong emission of the condensate occurs and can easily mask the GB signal. Furthermore, the incoherent reservoir might affect the Bogoliubov-like spectra of the excitations of the condensate. The resonant experiment done by Kohnle et al. in [10] is in stark contrast to the nonresonant pumping scheme, since the polariton condensate formation occurred in the absence of an incoherent reservoir and the collective excitations were created by a resonant trigger pulse.

In this Letter, we report on the direct observation of a PL signal of the GB together with a pronounced linearization of the dispersion of an expanding polariton condensate under nonresonant excitation. We verify the observation via polarization- and time-resolved experiments, which elucidate the origin of the observed features.

Details on the experimental setup and the sample used are given in the Supplemental Material [20].

In our experiment the pulsed pumping laser was focused to a diffraction-limited Gaussian shape of about $2 \mu \mathrm{m}$ in diameter. The PL intensity as a function of the excitation power for two linear and perpendicular polarization 

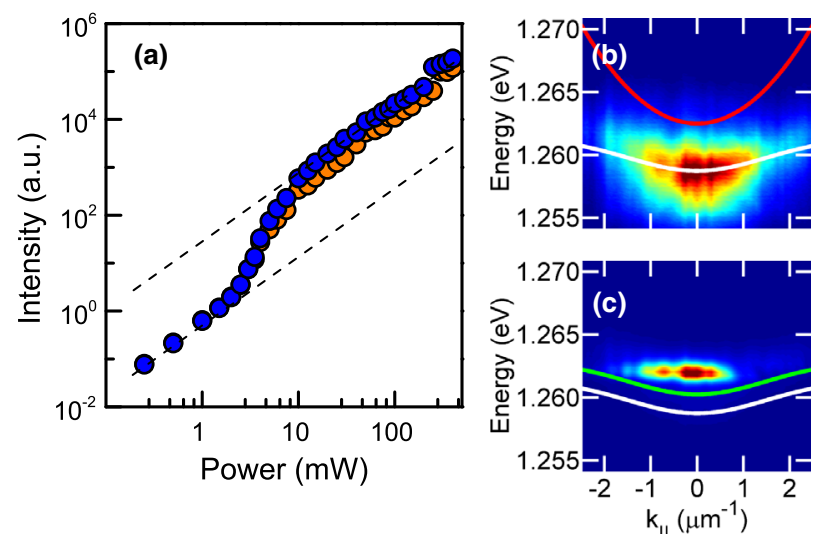

FIG. 1 (color online). (a) Power-dependent intensity curve of two linear polarizations, colinear to the condensate axis (blue circles) and cross-linear to the condensate (orange circles). (b) and (c) Time-integrated dispersions (b) below $\left(1 \mathrm{~mW}=0.1 P_{\text {th }}\right)$ the threshold and (c) slightly above the threshold $(5 \mathrm{~mW}=$ $\left.1.66 P_{\mathrm{th}}\right)$. LP branch (white line), blueshifted (green line), and bare-cavity mode (red line) dispersions are shown. The color scale is linear. The measurements have been done at the cavityexciton detuning of $-0.5 \mathrm{meV}$.

detections, colinear and cross-linear to the polarization axis of the polariton condensate, is shown in Fig. 1(a). The data are extracted from time-integrated data; hence, the values are averaged over many excitation pulses. At low excitation densities, the lower polariton (LP) branch dispersion is formed, as can be seen in Fig. 1(b). With a further increase of the excitation power, we observe a distinct nonlinear threshold in the intensity dependence on pumping shown in Fig. 1(a), consistent with the formation of a flat dispersion of a polariton condensate [Fig. 1(c)] at the threshold. This kind of dispersion is predicted [16] when exciting a polariton condensate by a small excitation spot. In our case, it is most likely caused by the localization of polaritons by the disorder potential. The condensation threshold is also manifested by a decrease of the linewidth of the measured emission, which drops down by a factor of 5 at threshold, and by a blueshift of the emission peak of about $3 \mathrm{meV}[20]$. The energy shift of the propagating condensate created nonresonantly is composed of the kinetic energy of polaritons, repulsive interactions within the condensate, and a term coming from the interaction of polaritons with the pump-spot-induced reservoir of noncondensed quasiparticles $[15,21]$. Hence, the condensate energy is shifted above the minimum of the bare cavity mode, while the strong coupling is preserved.

The microcavity polaritons are subject to a natural disorder potential, which originates from the width and composition fluctuations of the quantum wells and Bragg mirrors [22]. We observe polariton trapping by the disorder potential, as one can see in Fig. 2. Localization of polaritons in real space has been observed at moderate pump densities in our experiment. Bright spots of the luminescence located outside the pump area are visible in Fig. 2(a),
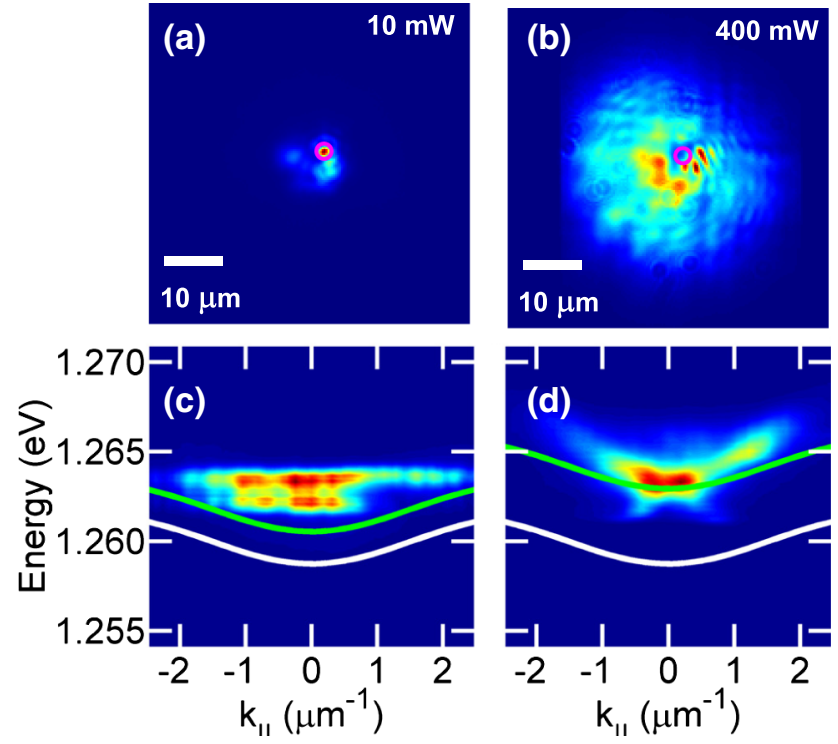

FIG. 2 (color online). (a) Real-space dispersion of localized polaritons at moderate densities. (b) Real-space distribution of the extended polariton cloud. (c) Corresponding momentum-space picture to (a). (d) Onset of linear dispersion at high pumping conditions. Line description is the same as in Fig. 1. The pump spot location is indicated as a pink circle in (a) and (b). The color scale is linear.

in agreement with the flat parts of the polariton dispersion observed in the reciprocal space [Fig. 2(c)] [23]. The most striking phenomena occur at higher polariton densities. First, we observed an evident spreading of the polariton cloud to distances much larger than the pump spot, see Fig. 2(b). This can be interpreted in terms of the ballistic flow of the polariton condensate pumped above the percolation threshold in a 2D disorder potential. Furthermore, a pronounced linearization of the polariton emission and clear evidence of the GB emission at negative energies (below the condensate emission energy) have been observed, as presented in Fig. 2(d). The GB becomes visible at pump densities around $20 P_{\text {th }}$ at different detunings and at different spot positions at the sample surface. The buildup of the GB luminescence could be attributed to the off-branch multiple-scattering processes in the polariton gas [7]. However, the intensity of the GB is strong, comparable to the $\mathrm{NB}$, and we detected a change in the dispersion curvature, indicating a collective phenomenon of mixing the NB and GB [10]. The linear spectrum and the appearance of the GB are fingerprints of collective Bogoliubov-like excitations of a propagating polariton fluid. Similar features can be induced resonantly in a propagating condensate in the supersonic regime in the presence of obstacles that disturb the flow [24,25]. Here, one can observe interference ripples in the real-space image [Fig. 2(d)]; this is a signature of nonradial propagation of scattered polariton waves [26]. In our sample, the magnitude of the disorder-potential fluctuations can be as high as 
$3 \mathrm{meV}$, comparable to the observed blueshift; hence, it is believed to be the source of excitations in an expanding polariton fluid, as it behaves as a static scattering landscape. The PL signal coming from ghost excitations is distributed on macroscopic distances, outside of the pump spot [20]. Additionally, one notes a slight anisotropy of the emission intensity distribution over the dispersion branches, which reflects a nonuniform disorder potential in the real space.

Let us now discuss the polarization characteristics of the emitted photons. One important property of polaritons is their spin-fine structure, inherited from excitons and photons. The optically active LPs have the spin projection $S= \pm 1$ on the growth-axis direction. Because of the anisotropic spin interactions, the minimization of the system energy favors the superposition of spin-up and spin-down polaritons at the condensation threshold, which results in a linear polarization buildup [27]. In our experiment, the nonlinear threshold is accompanied by an abrupt increase of the degree of linear polarization (DOLP) around $\mathbf{k}=0$, presented in Fig. 3(a) (where DOLP $=I_{\|}-I_{\perp} / I_{\|}+I_{\perp}$, and $I_{\|, \perp}$ are the intensities detected in two orthogonal linear polarizations). The linear polarization vector of the condensate has the same direction at each point in the real space. Our sample is disordered, which is why the polarization vector of the condensate is always pinned to one of the crystal axes [28,29]. For higher pumping levels, we observe a depinning of the polarization vector, which is manifested in a decrease of the total DOLP with respect to the pump power [30]. This data is extracted from the time-integrated measurements; the measured DOLP values are, thus, averaged over hundreds of time evolutions, resulting in the lower value of the DOLP compared to each individual realization [31]. Taking into
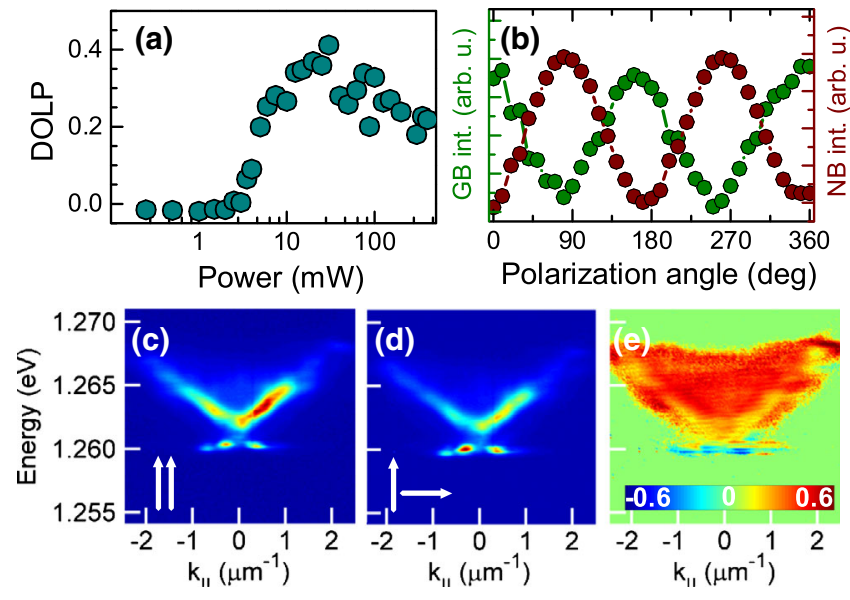

FIG. 3 (color online). (a) DOLP as a function of the pumping power. (b) Polarizations of the NB and the GB extracted from the PL map measurements. The values are normalized. (c) Colinear and (d) cross-linear dispersion with respect to the condensate polarization axis and (e) the corresponding DOLP map of dispersion at the detuning of $-0.5 \mathrm{meV}$. The DOLP scale is in the inset of (e). The intensity color scale is linear in (c) and (d). account the polarization properties of the excitations, NB and GB states are available for polaritons both colinear and orthogonal to the condensate polarization vector. However, considering the occupation process of the NB and the GB in our experiment, one should expect the NB to be polarized as the condensate and the GB signal to be orthogonal to it. This is due to the physical origin of the two coupled branches of excitations. While the NB is populated due to the nonzero effective temperature of the polariton gas, the GB is populated due to the depletion of the condensate by polariton-polariton scattering, similar to the off-branch scattered states observed at resonant pumping in Ref. [7]. According to the polarization selection rules [31,32], the scattering of linearly polarized polaritons preferentially produces polaritons having an orthogonal linear polarization, as a result of spin-dependent polariton-polariton scattering [20]. Indeed, our polarization-resolved measurements have shown the GB to be orthogonally polarized with respect to the condensate polarization axis. The results are shown in Fig. 3(b), where the signal from separate GBs and NBs as a function of polarization angle is shown, and Fig. 3(e), where we plot a DOLP map created from a direct overlap of the two dispersions from Figs. 3(c) and 3(d) and the calculation of the polarization degree for each pixel of the recorded data. A large area of a positive DOLP is observed for the NB, which comes from the background created by the full-time evolution of the signal after the pump pulse. More importantly, very distinct inversed polarization of the GB is clearly visible in the DOLP map. It is worth noting that while the optical nonlinearities of the cavity itself can generate an analogue of complex quantum fluid phenomena in the paraxial geometry [33], however, the large value of DOLP and linear polarization inversion for the GB is direct evidence of the polaritonpolariton scattering responsible for the population of the GB. Clearly, this cannot be an effect of the linear polarization splitting in the cavity, which could give, rather, a rise to the buildup of the circular polarization of emission [34].

All the data discussed above has been obtained in the time-integrated measurements. The main drawback of this kind of approach in experiments with pulsed excitation is a loss of the information about the complex dynamics of the system after each excitation pulse; in particular, it leads to the time averaging of the blueshift of the polariton dispersion branch. This has been addressed in Ref. [10], where the authors observed an overestimated speed of sound in the time-integrated picture as well as an asymmetry between the slopes of the NB and the GB. One can observe a similar asymmetry in our experiment, where the NB's slope is greater than that of the GB, see Figs. 3(c)$3(d)$. In order to rule out the artifacts in our dispersions that might arise due to the time integration, we have performed the time-resolved scanning of the measured dispersion of a propagating polariton condensate at the detuning of 

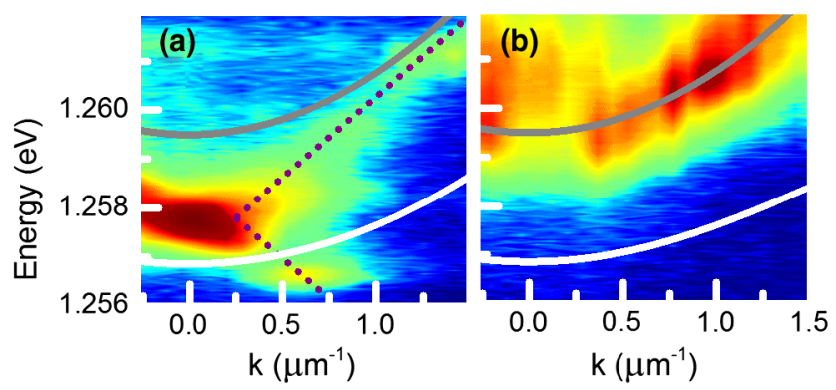

FIG. 4 (color online). (a) Dispersion snapshot at 66 ps of the time-resolved evolution of the polariton dispersion at the detuning of $-2 \mathrm{meV}$. Cavity mode and LP are shown by gray and white solid lines, respectively. The violet dotted lines are guides to the eye to highlight the similar slopes of the linear dispersion part. (b) Snapshot at the ultrashort times after the pulse arrival, presenting photon lasing in the weak coupling regime. The color scales are logarithmic.

$-2 \mathrm{meV}$, and we studied in detail the dynamics of the polariton luminescence after the pump pulse.

Approximately $50 \mathrm{ps}$ after the pump-pulse arrival we observe a simultaneous and distinct appearance of the positive and negative branches in the photoluminescence. We exclude here the apparatus effect, as the collected signal lasts much longer than the response resolution of the setup. A snapshot at $66 \mathrm{ps}$ after the pulse arrival is shown in Fig. 4(a). One can notice that the NB and the GB now have comparable slopes in this time-resolved picture. However, the extracted propagation velocity is still somewhat larger than the speed of sound calculated from the condensate blueshift from Fig. 4(a): the velocity taken from the slope of the time-resolved experiment is $v_{\text {slope }} \approx 1.95 \mu \mathrm{m} / \mathrm{ps}$ and is greater than the velocity of $v_{\text {blue }} \approx 1.45 \mu \mathrm{m} / \mathrm{ps}$ calculated from the blueshift at $\boldsymbol{k}=\mathbf{0}$, according to the definition $c=\sqrt{U / m_{\mathrm{pol}}}$, where $U$ is the blueshift and $m_{\mathrm{pol}}$ is the LP effective mass. The $v_{\text {slope }}$ corresponds to the polariton mean field energy of $U \approx 3.8 \mathrm{meV}$, being four times larger than the observed temporal blueshift. It has to be noted that the excitation scheme used here creates a condensate with a nontrivial spatial distribution of wave vectors [15]. Our case is far from the steady state of a static condensate; in this experimental configuration the pump pulse creates moving condensate, characterized by the outward coherent flow and disorder scattering of the polaritonic waves. This is why the condensate at zero wave vector is more likely to be created via scattering on multiple defect centers [35], resulting in a blueshift lower than that for a static condensate. We can also speculate on the spatially varying local Doppler shift of created excitations, which might be responsible for the observed distinct slopes, as the local condensate velocity is also added to the slope of the dispersion.

The observed emission of the condensate and the GB are present at the energy very close to, or even above, the energy of the cavity mode in our sample; this gives rise to the question whether these phenomena still occur in the strong coupling regime. In the time-resolved spectrum, right after the pump pulse arrival, we observe a strong emission corresponding to the bare-cavity mode at the time scale below the temporal resolution of our setup, as shown in Fig. 4(b). All the recorded dispersion features, including the linearized $\mathrm{NB}$ and the orthogonally polarized GB signal, occur later in time, where the weak coupling lasing has completely vanished. We conclude that these spectral features are characteristics of a polariton condensate formed in the strong coupling regime. In fact, in the time-resolved measurements we observe a transition from weak to strong coupling similar to the one reported in [36]. Furthermore, the signal related to the GB and the pronounced slope of the NB is clearly distinguishable from possible weakly coupled lasing from localized states, which may appear in their energetic proximity. The large distance spread of the emission cloud from the pump spot is achievable only for low-mass particles (the weak coupling lasing spot radius should evolve differently with pumping power [20,37]) and the depinning of the polarization vector of the condensate NB is exclusive for interacting particles, as it is a manifestation of strong coupling preservation [30].

We believe that we were able to observe very clearly the renormalization of the NB and the buildup of the GB partly because of the relatively modest quality factor of our sample, around 1000 [20], which was low enough to enable the efficient extraction of photons outside the cavity and high enough to preserve the conditions for the polariton condensate formation. Moreover, the intrinsic large disorder of the cavity enhanced the elastic scattering favorable for the formation of the condensate excitations.

In conclusion, we report on the first observation of a polariton ghost branch in a photoluminescence experiment under nonresonant excitation. Even though the system in our approach was far from equilibrium and from the steady-state regime, we have observed a distinct renormalization of the polariton dispersion characterized by the Bogoliubov-like normal and ghost branches. The origin of the observed dispersion branches has been identified based on the observed orthogonal linear polarizations of the branches and on the time-resolved dispersions, which show temporal symmetric slopes for the normal and ghost branches of the polariton condensate excitations.

We would like to thank Iacopo Carusotto for the critical reading of this manuscript and for valuable discussions. The authors acknowledge financial support from the bilateral project of Deutsche Forschungsgemeinschaft (Project LIEPOLATE) and the Polish Ministry of Science and Higher Education (Project No. DPN/N99/DFG/2010). The experiment was partially performed within the NLTK infrastructure, Project No. POIG.02.02.00-003/ 08-00. A. K. acknowledges financial support from the Russian Ministry of Education and Science (Contract No. 11.G34.31.0067). 
*Corresponding author. maciej.pieczarka@pwr.edu.pl

[1] T. Byrnes, N. Y. Kim, and Y. Yamamoto, Nat. Phys. 10, 803 (2014).

[2] I. Carusotto and C. Ciuti, Rev. Mod. Phys. 85, 299 (2013).

[3] J. Kasprzak, M. Richard, S. Kundermann, A. Baas, P. Jeambrun, J. M. J. Keeling, F. M. Marchetti, M. H. Szymańska, R. André, J. L. Staehli, V. Savona, P. B. Littlewood, B. Deveaud, and L. S. Dang, Nature (London) 443, 409 (2006).

[4] J. Fischer, I. G. Savenko, M. D. Fraser, S. Holzinger, S. Brodbeck, M. Kamp, I. A. Shelykh, C. Schneider, and S. Höfling, Phys. Rev. Lett. 113, 203902 (2014).

[5] F. Manni, K. G. Lagoudakis, R. André, M. Wouters, and B. Deveaud, Phys. Rev. Lett. 109, 150409 (2012).

[6] G. Nardin, G. Grosso, Y. Léger, B. Piętka, F. MorierGenoud, and B. Deveaud-Plédran, Nat. Phys. 7, 635 (2011).

[7] P. G. Savvidis, C. Ciuti, J. J. Baumberg, D. M. Whittaker, M. S. Skolnick, and J. S. Roberts, Phys. Rev. B 64, 075311 (2001).

[8] J. Zajac and W. Langbein, arXiv:1210.1455.

[9] L. Pitaevskii and S. Stringari, Bose-Einstein Condensation (Oxford University Press, New York, 2003).

[10] V. Kohnle, Y. Léger, M. Wouters, M. Richard, M. T. Portella-Oberli, and B. Deveaud-Plédran, Phys. Rev. Lett. 106, 255302 (2011).

[11] C. Schneider, A. Rahimi-Iman, N. Y. Kim, J. Fischer, I. G. Savenko, M. Amthor, M. Lermer, A. Wolf, L. Worschech, V. D. Kulakovskii, I. A. Shelykh, M. Kamp, S. Reitzenstein, A. Forchel, Y. Yamamoto, and S. Höfling, Nature (London) 497, 348 (2013).

[12] P. Bhattacharya, T. Frost, S. Deshpande, M.Z. Baten, A. Hazari, and A. Das, Phys. Rev. Lett. 112, 236802 (2014).

[13] F. Tassone, C. Piermarocchi, V. Savona, A. Quattropani, and P. Schwendimann, Phys. Rev. B 56, 7554 (1997).

[14] J. Schmutzler, T. Kazimierczuk, Ö. Bayraktar, M. Aßmann, M. Bayer, S. Brodbeck, M. Kamp, C. Schneider, and S. Höfling, Phys. Rev. B 89, 115119 (2014).

[15] M. Wouters, I. Carusotto, and C. Ciuti, Phys. Rev. B 77, 115340 (2008).

[16] M. Wouters and I. Carusotto, Phys. Rev. Lett. 99, 140402 (2007).

[17] S. Utsunomiya, L. Tian, G. Roumpos, C. W. Lai, N. Kumada, T. Fujisawa, M. Kuwata-Gonokami, A. Löffler, S. Höfling, A. Forchel, and Y. Yamamoto, Nat. Phys. 4, 700 (2008).

[18] M. Assmann, J.-S. Tempel, F. Veit, M. Bayer, A. RahimiIman, A. Löffler, S. Höfling, S. Reitzenstein, L. Worschech, and A. Forchel, Proc. Natl. Acad. Sci. U.S.A. 108, 1804 (2011).

[19] T. Byrnes, T. Horikiri, N. Ishida, M. Fraser, and Y. Yamamoto, Phys. Rev. B 85, 075130 (2012).

[20] See Supplemental Material at http://link.aps.org/ supplemental/10.1103/PhysRevLett.115.186401, which includes Refs. [26,31,32,38-46].

[21] P. Cilibrizzi, A. Askitopoulos, M. Silva, F. Bastiman, E. Clarke, J. M. Zajac, W. Langbein, and P. G. Lagoudakis, Appl. Phys. Lett. 105, 191118 (2014).
[22] V. Savona, J. Phys. Condens. Matter 19, 295208 (2007).

[23] D. Sanvitto, A. Amo, L. Viña, R. André, D. Solnyshkov, and G. Malpuech, Phys. Rev. B 80, 045301 (2009).

[24] I. Carusotto and C. Ciuti, Phys. Rev. Lett. 93, 166401 (2004).

[25] A. Amo, J. Lefrère, S. Pigeon, C. Adrados, C. Ciuti, I. Carusotto, R. Houdré, E. Giacobino, and A. Bramati, Nat. Phys. 5, 805 (2009).

[26] G. Christmann, G. Tosi, N. G. Berloff, P. Tsotsis, P. S. Eldridge, Z. Hatzopoulos, P. G. Savvidis, and J. J. Baumberg, Phys. Rev. B 85, 235303 (2012).

[27] F. P. Laussy, I. A. Shelykh, G. Malpuech, and A. Kavokin, Phys. Rev. B 73, 035315 (2006).

[28] J. Kasprzak, R. André, L. S. Dang, I. A. Shelykh, A. V. Kavokin, Y. G. Rubo, K. V. Kavokin, and G. Malpuech, Phys. Rev. B 75, 045326 (2007).

[29] Ł. Kłopotowski, M. D. Martín, A. Amo, L. Viña, I. A. Shelykh, M. M. Glazov, G. Malpuech, A. V. Kavokin, and R. André, Solid State Commun. 139, 511 (2006).

[30] J. Levrat, R. Butté, T. Christian, M. Glauser, E. Feltin, J.-F. Carlin, N. Grandjean, D. Read, A. V. Kavokin, and Y. G. Rubo, Phys. Rev. Lett. 104, 166402 (2010).

[31] T. Ostatnický and A. V. Kavokin, Superlattices Microstruct. 47, 39 (2010).

[32] T. Ostatnický, I. A. Shelykh, and A. V. Kavokin, Phys. Rev. B 81, 125319 (2010).

[33] J. Scheuer and M. Orenstein, Science 285, 230 (1999).

[34] P. Cilibrizzi, H. Ohadi, T. Ostatnicky, A. Askitopoulos, W. Langbein, and P. Lagoudakis, Phys. Rev. Lett. 113, 103901 (2014).

[35] M. Wouters and I. Carusotto, Phys. Rev. Lett. 105, 020602 (2010).

[36] E. Kammann, H. Ohadi, M. Maragkou, A. V. Kavokin, and P. G. Lagoudakis, New J. Phys. 14, 105003 (2012).

[37] H. Deng, G. Weihs, D. Snoke, J. Bloch, and Y. Yamamoto, Proc. Natl. Acad. Sci. U.S.A. 100, 15318 (2003).

[38] G. Roumpos, M. D. Fraser, A. Löffler, S. Höfling, A. Forchel, and Y. Yamamoto, Nat. Phys. 7, 129 (2011).

[39] J. M. Zajac, E. Clarke, and W. Langbein, Appl. Phys. Lett. 101, 041114 (2012).

[40] A. Baas, K. G. Lagoudakis, M. Richard, R. André, L. S. Dang, and B. Deveaud-Plédran, Phys. Rev. Lett. 100, 170401 (2008).

[41] E. Wertz, L. Ferrier, D. Solnyshkov, R. Johne, D. Sanvitto, A. Lemaître, I. Sagnes, R. Grousson, A. V. Kavokin, P. Senellart, G. Malpuech, and J. Bloch, Nat. Phys. 6, 860 (2010).

[42] I. A. Shelykh, Y. G. Rubo, G. Malpuech, D. D. Solnyshkov, and A. Kavokin, Phys. Rev. Lett. 97, 066402 (2006).

[43] D. Read, T. C. H. Liew, Y. G. Rubo, and A. V. Kavokin, Phys. Rev. B 80, 195309 (2009).

[44] S. Schumacher, N. H. Kwong, and R. Binder, Phys. Rev. B 76, 245324 (2007).

[45] P. Renucci, T. Amand, X. Marie, P. Senellart, J. Bloch, B. Sermage, and K. V. Kavokin, Phys. Rev. B 72, 075317 (2005).

[46] M. Romanelli, C. Leyder, J. P. Karr, E. Giacobino, and A. Bramati, Phys. Rev. Lett., 98, 106401 (2007). 